\title{
Modeling the Annual Costs of Postmenopausal Prevention Therapy: Raloxifene, Alendronate, or Estrogen-Progestin Therapy
}

\author{
C. DANIEL MULLINS, PhD, and ROBERT L. OHSFELDT, PhD
}

\begin{abstract}
OBJECTIVE: To estimate the annual cost and outcome impacts attributable to raloxifene, alendronate, and estrogen-progestin therapy as prevention therapies among postmenopausal women over the first 7 years of hormone replacement therapy (HRT).

METHODS: A budget-impact model was devised to compare the costs, benefits, and costs per event avoided for various postmenopausal therapies (raloxifene, alendronate, or estrogen-progestin combination therapy), compared to no intervention, taking into account the persistency rates. Net costs are direct medical costs attributable to treatments relative to no intervention. Net benefits are defined as the number of events avoided as a result of therapy. The main outcome measures are annual total net costs, net benefits, and costs per event avoided compared to no intervention among postmenopausal white women with intact uteri and normal baseline risks for osteoporotic hip or vertebral fractures, fatal or nonfatal myocardial infarction, and breast cancer. Data and model assumptions are based on clinical trial data and published retrospective studies.
\end{abstract}

RESULTS: The average annual net cost of therapy declines after the first year of therapy for all interventions, primarily due to discontinuation, and continues to decline over time due to savings in medical costs for events avoided. Net events avoided are greater for raloxifene than alendronate, but HRT use results in net harm. The cost per event avoided is lower for raloxifene than alendronate. Improved persistence improves the cost-effectiveness for both interventions. Sensitivity analyses indicate the model results are most sensitive to the assumed impact of raloxifene on coronary heart disease and breast cancer risk.

Alendronate as a prevention intervention is dominated by raloxifene under almost all model scenarios.

CONCLUSION: The annual cost of long-term postmenopausal prevention therapy is highest during the first few years of therapy. Long-term prevention does not provide a return on investment in fewer than 3 years, but savings in medical costs partially offset intervention costs after 2 years. For postmenopausal women, pharmacologic interventions with multiple prevention benefits tend to be more cost effective than interventions with a single source of health benefit.

KEYWORDS: Postmenopausal, Prevention, Cost-effectiveness, 0steoporosis

J Managed Care Pharm. 2003(9)2:150-58

Authors

C. DANIEL MULLINS, PhD, is an Associate Professor, Department of Pharmacy Practice and Science, School of Pharmacy, University of Maryland, Baltimore; ROBERT L. OHSFELDT, PhD, is a Professor, Department of Health Management \& Policy, College of Public Health, University of Iowa, Iowa City. (Ohsfeldt was formerly with Eli Lilly and Company.)

AUTHOR CORRESPONDENCE: C. Daniel Mullins, PhD, Associate Professor, Department of Pharmacy Practice and Science, School of Pharmacy, University of Maryland. 515 W. Lombard St., Room 262, Baltimore, MD 21201-1563. Tel: (410) 706-0879; Fax: (410)706-5394; Email: dmullins@rx.umaryland.edu

Copyright $\odot$ 2003, Academy of Managed Care Pharmacy. All rights reserved
$\mathrm{F}$ or any new pharmacological agent, it is difficult to assess the agent's economic impact on health care system costs based on clinical trial data alone. In such cases, cost modeling may be particularly useful in estimating the potential economic impact of a new agent. Cost models make use of the best available evidence about the clinical effectiveness of alternative agents and the resource consumption attributable to their use. A cost model can assist decision makers until more definitive information becomes available about outcomes and costs in actual clinical practice. Such analyses can also inform and structure subsequent prospective trials, leading to far better trial designs.

Traditionally, cost-effectiveness or cost-utility analyses of long-term prevention therapies compare the (discounted) lifetime costs of therapy to its lifetime clinical benefits, often summarized as incremental cost per quality-adjusted life-year (QALY) gained. ${ }^{1}$ However, this type of cost-effectiveness information often is either not used at all or given little weight in decisions about the formulary status of drugs. ${ }^{1-3}$ A number of possible explanations for this phenomenon exist, but a likely barrier to use of cost-effectiveness analysis is that formulary decision makers often have a shorter planning horizon than that employed in standard cost-effectiveness analyses. The decision to "invest" in prevention may be viewed as a capital budgeting issue, where data for the time-path of costs and benefits over a relevant planning horizon are needed to assess alternative investment options. However, the current standards for reporting results of cost-effectiveness analyses do not require or recommend reporting the time-path of costs and benefits. ${ }^{1}$ Thus, cost-effectiveness models that employ net present values for a 30-year or longer time horizon may not be considered relevant for these decision makers.

In contrast to lifetime models, in this paper, a medium-term cost-effectiveness model is developed using the perspective of a payer such as a managed care organization. Specifically, the model provides estimates of the annual costs and outcome impacts attributable to the use of alternative pharmaceutical agents for the primary prevention of osteoporosis compared to no drug therapy, over the first 7 years of therapy. In addition, the model departs from many cost-effectiveness analyses by accounting for the impact of early discontinuation of therapy observed in clinical practice, which provides a more realistic assessment of the potential costs and benefits of an intervention. The objective of this medium-term cost model is to estimate annual cost and outcome impacts attributable to raloxifene, alendronate and estrogen-progestin therapy as prevention therapies among postmenopausal women over the first 7 years of 
FIGURE 1 Tree Diagram Illustrating the Structure of the Model

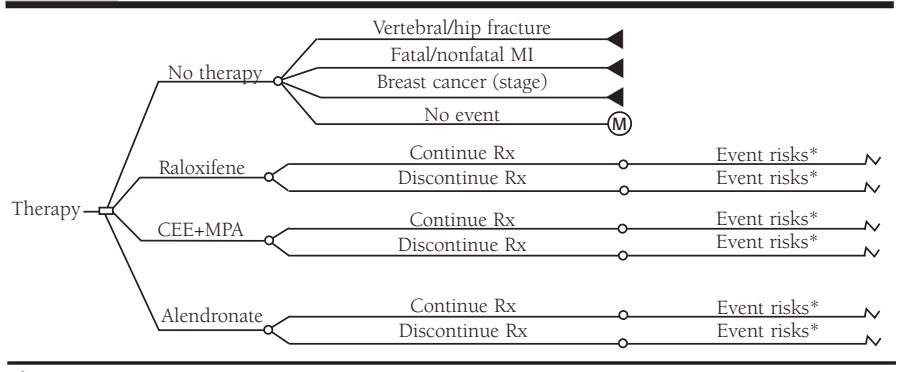

*The $\sim$ mark represents the same event branches illustrated for the no-drug-therapy arm.

therapy. The goal is to provide decision makers in managed care organizations with information needed to anticipate the budgetary impact of alternative prevention strategies, as well as an assessment of their potential benefits, within a time horizon relevant to the decision makers.

\section{Model Design}

The model compares osteoporosis prevention strategies using one of 3 prescription drugs or calcium and vitamin D supplementation only (no prescription drug intervention). The 3 prescription drug alternatives considered are (1) conjugated equine estrogens plus medroxyprogesterone acetate [CEE+MPA], a specific example of continuous-combined estrogen-progestin replacement therapy; (2) raloxifene hydrochloride, an agent within the class of drugs called selective estrogen receptor modulators (SERMs); and (3) alendronate, a bisphosphonate. In all cases, the prescription drug interventions include calcium and vitamin D supplementation. The model focuses on 3 main clinical outcomes: hip and vertebral fracture, fatal and nonfatal myocardial infarction (MI), and breast cancer. Direct medical costs and the number of model disease events (fractures, MIs, breast cancers) are estimated for each year for each of the 4 model arms. The differences in direct medical costs in the treatment arms and costs in the no-drug-intervention arm are defined as the net costs of therapy. Similarly, net benefits are defined simply as the number of clinical events avoided as a result of therapy.

The model results reported here focus on women who have not had a hysterectomy who initiate therapy at age 55 . As a base case, the populations considered consist of women representing a normal distribution of age-related baseline risks for the 3 outcomes of interest to avoid the complication of incorporating risk-assessment procedures and their costs into the model. However, the impact of "costless" risk stratification is assessed in alternative model scenarios. To simplify the model, a fixed population is analyzed (i.e., no disenrollment or new starts over the 7-year period).

To further simplify the model, the following clinical events are excluded: other osteoporotic fractures (e.g., wrist fractures), non-MI coronary heart disease (CHD) outcomes, uterine cancer, and venous thromboembolic events (VTE). The exclusion of other fracture outcomes biases the model results in favor of no drug intervention but may not bias comparisons across treatment arms significantly unless clinical effectiveness in preventing other fractures differs substantially across agents. Excluding non-MI CHD events (e.g., unstable angina, revascularization procedures not secondary to treatment for MI) may bias model results to the extent that either CEE+MPA or raloxifene affect non-MI CHD events. Excluding uterine cancer may favor $\mathrm{CEE}+\mathrm{MPA}$, although the bias should be negligible if, as generally assumed, the impact of CEE+MPA on uterine cancer risk is small. Other potential benefits (e.g., prevention of Alzheimer's disease) or risks (e.g., ovarian cancer) associated with CEE+MPA use are not accounted for in the model. The exclusion of VTE will bias the model in favor of both CEE+MPA and raloxifene. Since VTE risk increases with age, this bias will be larger in models focused on older postmenopausal women compared to models focused on younger postmenopausal women. For younger women, the exclusion of VTE will have a negligible impact on net costs. (For example, if CEE+MPA or raloxifene increases annual VTE risk from $1 / 1,000$ to 3/1,000, and the expected cost of VTE is $\$ 4,000$, the impact on net cost is $\$ 8$ per woman initiating therapy.)

A key determinant of costs and outcomes at the population level is the pattern of persistence of therapy. A prevention therapy that is initiated and then discontinued within a short period of time generates costs with little or no benefit. The mediumterm model presented here differs from many traditional costeffectiveness models of long-term prevention therapies in that the impact of early discontinuation of therapy on costs and outcomes is evaluated.

The general model structure is illustrated in Figure 1. For each of the 4 interventions (including no drug intervention), in each period, every woman faces some risk of an event: fracture (vertebral or hip), MI (fatal or nonfatal), or breast cancer (differentiated by stage at diagnosis). If no event occurs, the woman moves to the next period to face the risks again. If an event occurs, the process ends with an anticipated stream of costs in the current and subsequent years. In each of the prescription drug intervention arms (raloxifene, CEE+MPA, alendronate), annual event risks are altered by therapy. [To save space in the figure, the branch with the w mark represents the same event branches illustrated for the no-drug-therapy arm.] For the intervention arms, the full impact of therapy on event risks over time occurs only if a woman who initiates therapy remains persistent on therapy. If therapy is discontinued, she begins to revert to the event risks associated with no drug therapy.

As with any model, the specific effects of therapy on costs and outcomes are not known with certainty. This is particularly true for a new therapeutic option for which data are limited. A basecase model scenario is specified using reasonable estimates of the potential effectiveness of each therapy. To address uncertainty about the assumptions regarding the effects of therapy, alternative model scenarios are evaluated in sensitivity analyses. 


\section{Clinical Effectiveness Assumptions}

The clinical effectiveness assumptions, including all efficacy and safety assumptions, are summarized in Table 1. All assumptions about percent risk reduction are rounded to the nearest multiple of 5. The specific components of model assumptions are described in greater detail below.

Vertebral/hip fracture. Evidence of vertebral fracture prevention efficacy for raloxifene arises from the Multiple Outcomes of Raloxifene Evaluation (MORE) study, a placebocontrolled randomized clinical trial (RCT) with vertebral fracture as a primary endpoint. ${ }^{4}$ Vertebral fracture prevention efficacy assumptions for alendronate are taken from the Fracture Intervention Trials (FIT). ${ }^{5}$ In addition to these data, estimates of fracture efficacy after one year of therapy have been reported for both raloxifene ${ }^{6}$ and alendronate. ${ }^{7}$ These estimates are based on reported clinical vertebral fractures in MORE and FIT, respectively. Osteoporosis trials usually focus on vertebral fractures as a primary endpoint and thus are not powered statistically to detect differences in incident hip fractures between treatment and placebo groups. Nonetheless, in FIT, a statistically significant treatment effect for hip fracture risk reduction was observed for alendronate, but only in women with prior osteoporotic fractures. However, the base-case model scenario assumes the same relative reduction in hip fracture risk applies to women with low bone mass but no prior fractures using alendronate. No statistically significant differences in hip fracture incidence were observed in the MORE study. However, the treatment difference in vertebral bone mineral density (BMD) only accounts for (in statistical terms) about one third of the actual vertebral fracture rate treatment difference observed in MORE.,10 This fact, together with the fact that raloxifene increases total hip BMD ${ }^{11}$ suggests that raloxifene is likely to have some clinical benefit in terms of reducing hip fracture risk. Nonetheless, to be conservative, the base-case model scenario assumes no fracture prevention efficacy for raloxifene at the hip.

Until recently, no evidence for the fracture prevention efficacy of CEE+MPA from a large, placebo-controlled RCT was available. Estimates from observational studies were summarized in a report produced by the National Osteoporosis Foundation. ${ }^{8}$ However, given the potential for "prevention bias" in observational studies, such estimates may overstate CEE+ MPA's true fracture prevention efficacy. In the model, fracture efficacy assumptions for CEE+MPA are based on data from the recently halted estrogen-progestin therapy RCT conducted as part of the Women's Health Initiative (WHI), which reported a $33 \%$ reduction in both hip and vertebral fracture rates associated with CEE+MPA use over 5 years. ${ }^{12}$

Fatal/non-fatal MI. The data collected in MORE included CHD events as a secondary endpoint. Since the primary endpoint in MORE was vertebral fracture among women with osteoporosis, women participating in the study represented an approximately normal age-related distribution of $\mathrm{CHD}$ risks at baseline. Over the entire sample $(\mathrm{N}=7,705)$, use of raloxifene

\begin{tabular}{|c|c|c|c|c|}
\hline & & CEE+MPA & Raloxifene & Alendronate \\
\hline \multicolumn{5}{|l|}{ Fracture (Fx) } \\
\hline Hip Fx & $\begin{array}{l}(\mathrm{Y} 1) \\
(>\mathrm{Yl})\end{array}$ & $\begin{array}{l}-18 \% \\
-35 \%\end{array}$ & $\begin{array}{l}0 \\
0\end{array}$ & $\begin{array}{l}-25 \% \\
-50 \%\end{array}$ \\
\hline Vertebral Fx & $\begin{array}{l}\text { (Yl) } \\
(>\mathrm{Yl})\end{array}$ & $-45 \%$ & $\begin{array}{l}-65 \% \\
-50 \%\end{array}$ & $\begin{array}{l}-60 \% \\
-50 \%\end{array}$ \\
\hline Breast Cancer & $\begin{array}{l}(\mathrm{Y} 1) \\
(\mathrm{Y} 2-4) \\
(>\mathrm{Y} 4)\end{array}$ & $\begin{array}{c}0 \\
0 \\
+25 \%\end{array}$ & $\begin{array}{c}0 \\
-55 \% \\
-55 \%\end{array}$ & $\begin{array}{l}0 \\
0 \\
0\end{array}$ \\
\hline$\overline{\mathrm{CHD}}$ & $\begin{array}{l}(\mathrm{Y} 1) \\
(\mathrm{Y} 2) \\
(>\mathrm{Y} 2)\end{array}$ & $\begin{array}{c}+75 \% \\
+15 \% \\
0\end{array}$ & $\begin{array}{c}0 \\
0 \\
-15 \%\end{array}$ & $\begin{array}{l}0 \\
0 \\
0\end{array}$ \\
\hline
\end{tabular}

(60 mg) was associated with an $18 \%$ reduction in the risk of CHD events (95\% CI, 0.56, 1.22) over 4 years. A post-hoc analysis of a subset of 1,035 women at increased risk for cardiovascular disease at baseline found that use of raloxifene was associated with a 33\% reduction in the risk of cardiovascular disease events $(95 \% \mathrm{CI}, 0.37,1.19)$ over 4 years. There was no evidence of an "early" increase in risk in either sample. ${ }^{13}$

Neither of these point estimates is statistically significant. However, using the impact of raloxifene on serum cholesterol established in $\mathrm{RCTs}^{14}$ and the Coronary Heart Disease Policy Model to estimate the impact of therapy on the risk of a CHD event results in an estimated risk reduction of $14 \%$, which is similar to the $18 \%$ point estimate from the full sample in MORE. The literature suggests at least a 1-year lag in modeling the onset of effect of LDL cholesterol reduction on CHD event risk reduction. ${ }^{15}$ To be conservative, the base-case model scenario assumes a 15\% reduction in CHD risk after a 2-year lag in onset of effect for raloxifene.

The WHI study results showed a 29\% increase in the risk of CHD events associated with CEE+MPA use over 5 years. Findings reported by year of follow-up indicated point estimates of a $78 \%$ increase in risk during the first year of therapy, a 15\% increase during the second year, with approximately no difference thereafter. The finding of "early" CHD harm in WHI is consistent with the Heart and Estrogen/Progestin Replacement Study (HERS) ${ }^{16}$ finding of a statistically significant $50 \%$ increase in CHD risk during the first year of therapy. A reanalysis of the HERS data concluded that the use of CEE+MPA was associated with net CHD harm in terms of survival over 5 years. ${ }^{17}$ Other studies also suggest the potential for an increase in CHD risk associated with the initiation of HRT. ${ }^{18}$ Thus, the base-case model scenario assumes "early" harm for CEE+MPA during the first 2 years of therapy with no effect thereafter. The model assumes alendronate provides no CHD benefits or risks.

Breast cancer. Breast cancer was another secondary end- 


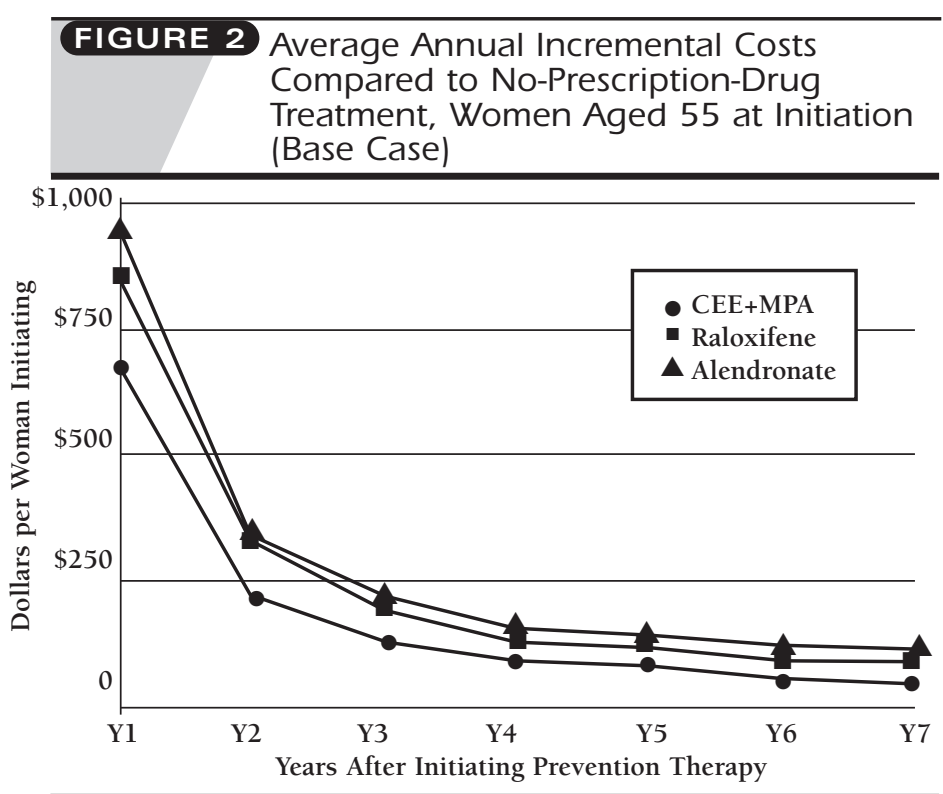

point in MORE. Participants in MORE represented an approximately normal age-related breast cancer risk distribution at baseline. Data from the MORE study found a $76 \%$ reduction in breast cancer incidence over 3 years of therapy. ${ }^{19}$ However, since the MORE study focused on an older osteoporosis treatment population (mean age at randomization of 65 years), generalizing its results to a younger osteoporosis prevention population (age of 55 years) may not be appropriate. To address this issue, data from pooled placebo-controlled clinical trials of raloxifene, similar to that reported by Jordan and colleagues ${ }^{20}$ but updated to include 3 years of follow-up data, were analyzed after stratification by age at randomization (under age 65, age 65 or older). ${ }^{21}$ The under-65 sample includes 3,865 patients taking either $60 \mathrm{mg}$ or $120 \mathrm{mg}$ of raloxifene and 1,841 patients taking placebo, with a mean age at randomization of 57.2 years. After 36 months of therapy, the incidence of all breast cancers was 5.2 per 1,000 in the raloxifene group, compared to 12.2 per 1,000 in the placebo group $(\mathrm{RR}=0.43 ; 95 \% \mathrm{CI}, 0.22,0.82 ; P=0.02)$. Based on these data, the base-case model scenario assumes no change in breast cancer risk attributable to raloxifene in the first year of therapy; after one year, the risk is assumed to decline by 55\% among women who are aged 55 years at initiation.

The WHI study found a 26\% increase in the relative risk of breast cancer associated with CEE+MPA use over 5 years. Trend analysis suggested that most of this increase in risk occurred after 3 years of therapy (point estimates for relative risk were 1.73 in year 4 and 2.64 in year 5). In the HERS study, there was a 38\% higher incidence of breast cancer in the CEE+MPA group than in the placebo group over the course of the 5-year study, though the difference was not statistically significant. ${ }^{22}$ These findings are consistent with a recent re-analysis of data from 51 observational studies, which concluded that each year of postmenopausal HRT increases the risk of breast cancer by $2.3 \%$. This translates into a $17 \%$ increase over 7 years of therapy. ${ }^{23,24}$ The base-case model scenario assumes the use of CEE+MPA increases the relative risk of breast cancer risk by $25 \%$ after 3 years of therapy. Alendronate is assumed to have no impact on breast cancer risk.

\section{Therapy Persistence Rates}

The assumed persistence rates for CEE+MPA are taken from Ettinger et al., ${ }^{25}$ extrapolated from 3 to 7 years. About 4 of 5 postmenopausal women who initiated CEE+MPA were found to have discontinued therapy within 3 years. The base-case model scenario assumes persistence rates (i.e., the percentage of women who have not discontinued therapy) across all 3 therapy arms are identical to the rate assumed for CEE+MPA. However, a recent analysis of data from a large managed care organization found that discontinuation rates at one year after initiation were higher for estrogen-progestin therapy compared to raloxifene. ${ }^{26}$ The base model assumes that $41 \%$ of patients are persistent at the end of the first year and 26\%,19\%, 17\%, 15\%, $14 \%$, and $13 \%$ are persistent at the end of the second through seventh year, respectively. Alternative scenarios are used to evaluate the impact of discontinuation rate assumptions.

\section{Costs}

The annual cost of each drug therapy is assumed to be the average wholesale price (AWP) as of October 1, 2000, for the recommended daily dosage times 365 plus the cost of one physician visit per year. ${ }^{27}$ To assess the costs of managing side effects or treating adverse events of therapy, estimates of resource utilization associated with side effects of HRT, ${ }^{28-33}$ alendronate, ${ }^{33-34}$ and raloxifene ${ }^{35}$ are combined with estimates of costs of resources (using Medicare payment rates) ${ }^{34}$ Most of the costs of side effects are assumed to occur during the first year of therapy, and all side-effect costs are assumed to end with discontinuation of therapy. Specifically, the side-effect costs used for years 1,2 , and 3 and beyond in the model are $\$ 200, \$ 100$, and $\$ 25$ for estrogen-progestin; $\$ 100, \$ 0$, and $\$ 0$ for alendronate; and $\$ 25, \$ 15$, and $\$ 0$ for raloxifene.

No direct estimates from RCTs of the cost impact of raloxifene or the alternative therapies are available. The model makes use of estimates of event costs in the literature to assess potential costs averted by therapy. Estimated direct medical costs attributable to hip fracture, fatal MI, and nonfatal MI are from the former Congressional Office of Technology Assessment. ${ }^{35}$ Estimated direct medical costs of vertebral fractures are from the National Osteoporosis Foundation. ${ }^{8}$ Estimated annual direct medical costs of treating breast cancer for 4 years postdiagnosis, by stage at diagnosis, are from Legoretta et al. ${ }^{36}$ Costs reported in the literature were converted to 2000-equivalent dollars using the medical care component of the consumer price index. Cumulative costs estimates are not discounted to their present value, though it should be noted that discounting has less impact over the medium-term period addressed in the model compared to a traditional lifetime model. 


\section{Results}

\section{Net Costs}

Net costs are defined as costs attributable to treatments relative to no drug intervention and consist of 3 components: (1) pharmacy costs, (2) cost of managing side effects or minor adverse events attributable to therapy, and (3) savings or costs associated with events (fractures, fatal/nonfatal MIs, or breast cancers) avoided by or attributable to therapy.

The base-case model scenario suggests that annual net costs per woman age 55 initiating raloxifene average about $\$ 860$ during the first year of therapy, decline to about $\$ 330$ per woman by the second year, and further decline to about $\$ 90$ per woman by the seventh year of therapy (Figure 2). Most of this decline is due to early discontinuation of therapy. Annual net costs per woman initiating CEE+MPA are expected to average about $\$ 682$ during the first year, declining to about $\$ 50$ by the seventh year. Again, much of this decline is due to early discontinuation. For alendronate, the annual incremental costs per woman initiating therapy are expected to average about $\$ 950$ during the first year, declining to about $\$ 110$ by the seventh year. (The U.S. Food and Drug Administration-approved daily dose for alendronate as a prevention therapy is $5 \mathrm{mg}$, but the fracture prevention efficacy assumptions in the model are from the FIT studies, where the daily dose was primarily $10 \mathrm{mg}$. Cost estimates are similar for both the $5 \mathrm{mg}$ and $10 \mathrm{mg}$ daily dose.) Again, much of this decline is due to early discontinuation of therapy, as well as reduced side-effect costs associated with "biased" discontinuation. That is, those experiencing side effects resulting in resource utilization are assumed to be more likely than others to discontinue therapy.

\section{Net Benefits}

Net benefits are defined as the difference in the number of model events (hip/vertebral fracture, fatal/nonfatal MI, breast cancer) for women initiating each therapy compared to the number of events among women in the no-drug-therapy group (i.e., net events avoided).

Over the first 3 years of raloxifene therapy, the base-case model scenario suggests an expected reduction of about 1.8 events per 1,000 women initiating therapy, increasing to 4.0 events per 1,000 initiating therapy after 7 years (Figure 3 ). The estimated difference in events after 7 years is about 0.7/1,000 for alendronate. These estimated benefits are modest in large part due to the assumed high rate of early discontinuation of therapy, as well as the 7-year focus of the model. The outcome effect for alendronate is particularly small because (a) it only reflects the risk of hip and vertebral fracture in the model and (b) the risk of hip fracture is quite small over a 7-year period in a cohort of women aged 55 years at initiation of therapy.

In contrast, the high assumed rate of discontinuation exacerbates the "early" CHD harm associated with CEE+MPA use. Women initiating therapy are exposed to these early risks, but too few remain on therapy to attain the benefits of fracture prevention.
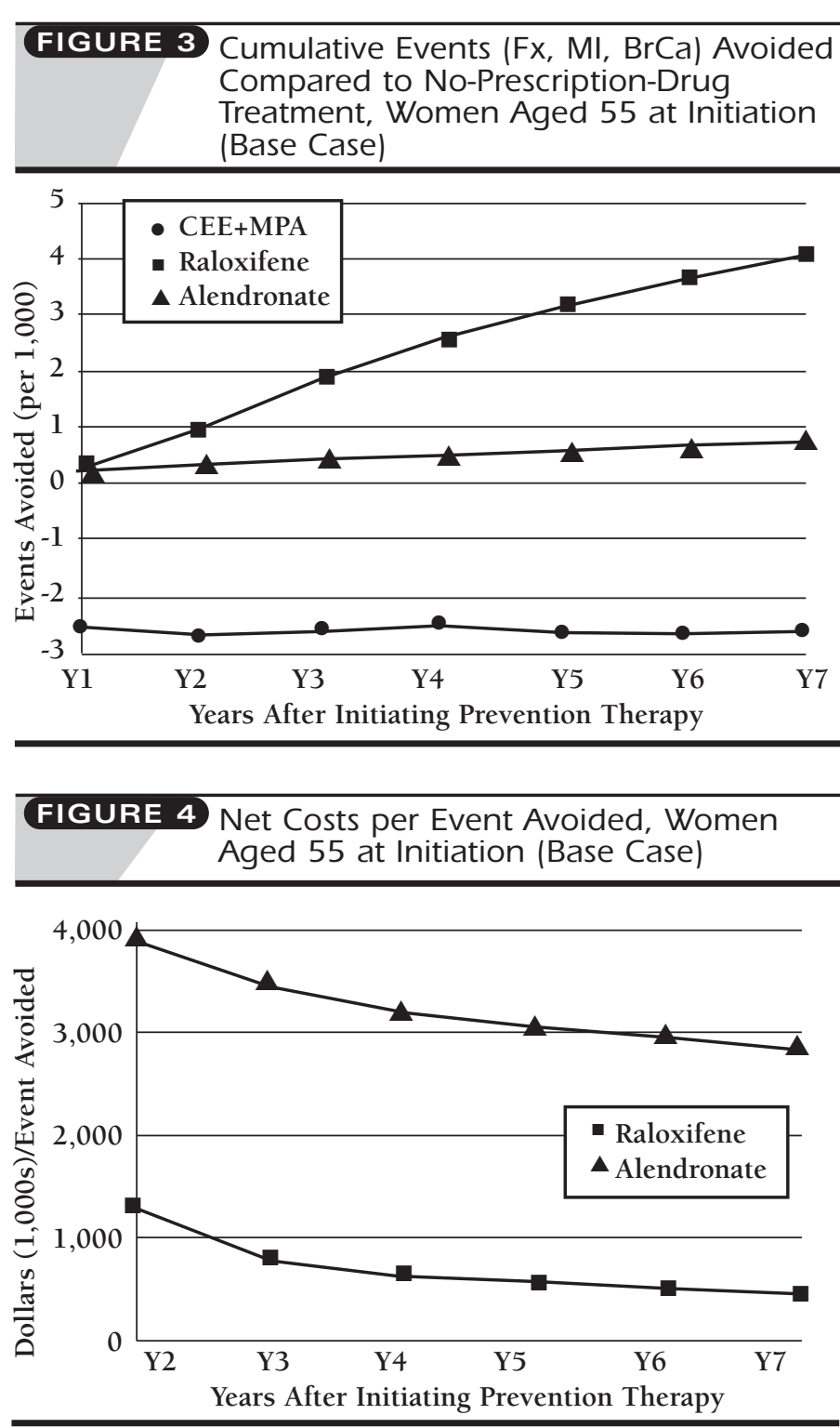

Furthermore, the relative few who remain on therapy for more than 4 years face an increase in breast cancer risk. As a result, CEE+MPA is associated with cumulative net harm (negative events avoided) in all 7 years.

\section{Cost-Effectiveness}

A cost-effectiveness ratio can be defined as the cumulative net costs of a therapy relative to its cumulative net benefits.

For CEE+MPA, cost-effectiveness is not a relevant issue in the base-case scenario due to estimated net harm. For both raloxifene and alendronate, the costs per event avoided during the first 2 years of therapy are substantial, as few of the benefits of longterm prevention accrue in just 2 years (Figure 4). For raloxifene, the costs per event avoided begin to decline after 2 years as more events are avoided. For alendronate, the decline in cost per event 


\section{TABLE 2 Sensitivity of Cost/Event-Avoided Estimates to Key Model Assumptions}

\begin{tabular}{lc}
\hline Therapy/Model Scenario & $\begin{array}{c}\text { Cost/Event } \\
\text { Avoided (\$Ks) }\end{array}$ \\
\hline Raloxifene Versus No Therapy (Base Case) & 455 \\
Hip Fx efficacy = 0.7 x alendronate efficacy & 425 \\
No CHD risk decrease (RR=1.0, Y1-7) & 600 \\
Higher CHD risk reduction (RR=.65, Y3-7) & 350 \\
Lower BrCa risk reduction (RR=.73, Y3-7) & 675 \\
No BrCa risk reduction (RR=1.0) & 1,350 \\
No CHD and no BrCa risk reduction (RR=1.0) & 4,180 \\
Population 2x normal fracture risk & 405 \\
Population 2x normal CHD risk & 380 \\
Population 2x normal breast cancer risk & 266 \\
Improved persistence (1.33 x CEE+MPA rate) & 405 \\
\hline CEE+MPA Versus No Therapy (Base Case) & Net Harm \\
No CHD risk increase (RR=1.0, Y1-7) & 3,925 \\
Pre-WHI presumed CHD benefit (RR=.75, Y3-7) & 640 \\
No breast cancer risk increase (RR=1.0, Y1-7) & net harm \\
Population 2x normal fracture risk & net harm \\
Population 2x normal CHD risk & net harm \\
Population 2x normal breast cancer risk & net harm \\
\hline Alendronate Versus No Therapy (Base Case) & 2,850 \\
AWP = risedronate AWP & 2,440 \\
Take 10 mg every other day (efficacy = QD) & 1,605 \\
Higher GI side-effect costs ( $\$ 600$, Y1) & 3,705 \\
Population 2x normal fracture risk & 1,440 \\
Population 3x normal fracture risk & 970 \\
Improved persistence (1.33 x CEE+MPA rate) & 2,720 \\
\hline &
\end{tabular}

avoided is not as substantial, since alendronate only reduces the risk of fracture outcomes in the model and is assumed to have no impact on CHD or breast cancer risk.

In the base-case scenario, the cost per event avoided over the first 7 years of therapy for raloxifene is about $\$ 455,000$ compared to about $\$ 2.9$ million per event avoided for alendronate. The impact of specific base-case model assumptions is assessed though a series of one-way sensitivity analyses (Table 2).

For raloxifene, the key area of sensitivity in the model relates to the assumed impact of therapy on the risk of breast cancer. In a model scenario where the use of raloxifene has no impact on breast cancer incidence, the cost per event avoided is about 3 times higher than in the base-case scenario. The assumed impact on CHD risk also affects model results. Compared to the base-case, the cost per event avoided increases by $30 \%$ if raloxifene does not reduce CHD risk but decreases by $23 \%$ if the magnitude of the risk reduction is as large as 35\% (similar to the point estimate for the high-risk subgroup in MORE). Improved persistence, as suggested by the results of the Kayser, Ettinger, and Pressman study, ${ }^{26}$ would improve cost-effectiveness, but not dramatically so. The population targeted for intervention also has some impact-among women at 2 times the normal age- related risk for breast cancer, the cost per event avoided falls to $\$ 266,000$ over 7 years.

In contrast, for CEE+MPA, virtually any model scenario with an assumption of significant "early" CHD harm (25\% or more relative risk increase during year 1) produces an estimate of cumulative net harm over 7 years of therapy. If CEE+MPA is assumed to neither increase nor decrease CHD risk, the estimated cost per event avoided is $\$ 3.9$ million. As had been assumed prior to the WHI findings, if CEE+MPA use was associated with a $25 \%$ reduction in CHD risk (after a 2-year lag) with no early harm, cost per event avoided would have been $\$ 640,000$ over 7 years.

For alendronate, an area of sensitivity is drug acquisition costs. If risedronate is considered clinically equivalent to alendronate, using its lower AWP reduces the cost per event avoided for bisphosphonate therapy to $\$ 2.4$ million. If alendronate $10 \mathrm{mg}$ is administered every other day as a prevention (versus treatment) therapy, with no adverse impact on efficacy, cost per event avoided falls to $\$ 1.6$ million. If the population targeted for alendronate therapy is at 2 times the normal age-related risk for osteoporotic fracture, its cost-effectiveness improves by $50 \%$ to $\$ 1.4$ million per event avoided, and improves to $\$ 970,000$ for a population at 3 times the normal fracture risk.

\section{Discussion}

It should be noted that the model presented here was submitted for publication prior to the publication of findings from the estrogen-progestin therapy component of the Women's Health Initiative. In revising the manuscript for publication, the basecase model assumptions for estrogen-progestin therapy were modified to reflect the WHI findings. The results imply net harm associated with CEE+MPA therapy under almost all scenarios. Thus, CEE+MPA should not be considered as a longterm prevention strategy. This implication is consistent with the recent recommendation by the U.S. Preventive Services Task Force to avoid using estrogen-progestin as a prevention therapy for healthy postmenopausal women. ${ }^{37}$

The analyses undertaken in this study are technically complex and should be interpreted in the context of the assumptions used in the models. The model employed here presents estimates of direct medical costs per "event avoided." As such, the reported cost-effectiveness estimates are not comparable to most published cost-effectiveness ratios for long-term prevention therapies. Further, it would be inappropriate to construct a "league table" comparing these "cost per event avoided" measures to the incremental "cost/QALY" measures in the literature. First, the model attempts to account for the practical challenge of persistence on long-term therapy by using therapy discontinuation assumptions based on experience in clinical practice. ${ }^{18}$ Most published models either assume complete persistence or use discontinuation rates from clinical trials. The use of more realistic discontinuation assumptions reduces the cost-effectiveness of all interventions relative to no intervention. Second, as 
a "medium-term" model, all future benefits of therapy over the remainder of life are not captured. Finally, given the payer perspective of the study, the model does not account for any indirect cost savings attributable to drug therapy.

Some indication of the differences between the results reported here and results from a more traditional cost-utility analysis (CUA) is provided by reference to a CUA by Armstrong and colleagues ${ }^{38}$ comparing raloxifene and HRT to no therapy over a life-cycle time horizon. This pre-WHI analysis assumes a 44\% reduction in CHD risk for HRT users as a base case, but a "CHD-neutral" scenario also is reported. (The potential for early CHD harm is not addressed.) In a model with HRT assumed to be "CHD neutral," the incremental cost per QALY over a lifetime horizon for HRT versus no therapy is $\$ 8,500$, compared to the estimate of $\$ 3.9$ million per event avoided over 7 years for a CHD-neutral scenario reported here. In addition to the shorter time horizon, our substantially higher cost-effectiveness estimate results from the high rate of discontinuation incorporated into the analysis. (Armstrong and colleagues assume no discontinuation of therapy.) Armstrong and colleagues also report a lifetime incremental cost per QALY of $\$ 9,820$ for raloxifene versus no therapy in their base-case scenario (normal breast cancer risk). This estimate falls to $\$ 4,100 /$ QALY for a population of women at 3 times normal age-related breast cancer risk and \$1,900/QALY among women at 6 times normal risk. Bisphosphonates were not considered as a prevention option in the analysis reported by Armstrong and colleagues.

Published economic evaluations of bisphosponates tend to focus on the treatment of established osteoporosis, not prevention. ${ }^{39}$ Any broad-based osteoporosis prevention intervention using a prescription drug will tend to have high costs relative to benefits, especially over a 7-year time horizon, if the drug only prevents osteoporosis. Costs per event avoided for both raloxifene and alendronate in "fracture-only" models are estimated at more than $\$ 2$ million over 7 years. Even in a scenario where CEE+MPA is "CHD neutral" its cost per event avoided also exceeds $\$ 2$ million.

Indeed, most of the past economic evaluations of HRT as a broad-based postmenopausal prevention strategy that have found incremental costs per QALY less than $\$ 25,000$ were highly dependent on a presumed substantial CHD risk-reduction benefit. ${ }^{40}$ Thus, the promise of HRT as a cost-effective, broadbased postmenopausal prevention strategy was reliant on a presumption of a broad spectrum of clinical benefits. Although WHI has not yet evaluated all of the putative clinical benefits of long-term HRT (e.g., prevention of Alzheimer's disease), one of the most significant presumed benefits from a cost-effectiveness prospective- $\mathrm{CHD}$ risk reduction-now appears to be unsupportable. On the other hand, if SERMs such as raloxifene or others currently in development provide a spectrum of clinical benefits, as much of the available data suggest, they may prove to be more cost effective as broad-based prevention strategies than drug interventions with a single source of clinical benefit.

\section{Limitations}

The outcome measure used in the model is a simple metric of "clinical events avoided." Although aggregations of occurrences of clinical events with substantially different impacts on healthrelated quality of life (HR-QOL) are commonly used as primary endpoints in clinical trials (e.g., cardiovascular disease "events" avoided), in this case, vertebral fracture seems unlikely to have an impact on HR-QOL as substantial as hip fracture, fatal or nonfatal MI, or breast cancer. If an arbitrary weight with a value less than unity (e.g., 0.2) is assigned to a vertebral fracture when determining net events avoided, the impact is to decrease estimated net events avoided for both raloxifene and alendronate, thereby further increasing estimated costs per event avoided. However, given the similarity in assumed vertebral fracture prevention across therapies, the estimated differences in cost-effectiveness across therapies are not affected significantly.

As noted, the model incorporates a number of simplifying assumptions, including the exclusion of several potentially relevant clinical considerations. Recently reported data highlight some additional clinical considerations that may warrant greater attention in any future analysis. First, the model reported here does not include stroke as an outcome. However, the WHI data indicated that CEE+MPA therapy was associated with a $41 \%$ increase in the incidence of stroke over 5 years. In contrast, data from MORE indicate a potential reduction in stroke risk associated with raloxifene use. ${ }^{14,41}$ Second, the WHI data indicated that CEE+MPA therapy was associated with a 37\% reduction in risk for colorectal cancer. Incorporating a colorectal cancer benefit into a decisionmodel-based economic evaluation would partially offset the CHD and stroke harm associated CEE+MPA use. However, CEE+MPA still would be predicted to cause net harm except, perhaps, in a population at well above average risk for colorectal cancer and well below average risk for CHD and stroke.

\section{Conclusion}

For a variety of reasons, lifetime cost-effectiveness analyses are seldom used by managed care organizations when making decisions about reimbursement policies for specific pharmaceutical agents. Providing managed care decision makers with information about the time-path of cost and outcome effects of a new pharmaceutical agent may prove to be more useful than a summary of net present values, especially for models with a long time horizon. Such details can assist in assessments of potential budget impact and "pay-back" periods for potential investments in long-term prevention initiatives.

The example of a medium-term model presented in this paper focuses on alternative osteoporosis prevention strategies. Overall, in this example that focuses on alternative osteoporosis prevention strategies, the model results suggest that raloxifene provides greater cost-effectiveness than alendronate for women initiating therapy at age 55 over the first 7 years of therapy, under most model scenarios. The estimated cost-effectiveness for raloxifene is sensitive to assumptions about the magnitude 
of breast cancer risk reduction associated with therapy but remains more cost effective than alendronate as long as it provides clinical benefits beyond fracture reduction.

In a scenario where the use of raloxifene has no effect on the risk of developing breast cancer and no effect on CHD risk, alendronate provides a lower cost per event avoided than raloxifene. However, at several million dollars per event avoided, prevention with either drug under a "fracture benefit only" scenario generally would be considered cost prohibitive, at least over the time horizon examined. In general, a broad-based prevention intervention using a prescription drug that provides a spectrum of clinical benefits is more likely to be cost effective than a narrow-spectrum prescription drug intervention.

\section{ACKNOWLEDGMENTS}

Paula Funk Orsini and Sheila Weiss, from the University of Maryland School of Pharmacy, contributed to the development of an early version of the model used in this analysis.

\section{DISCLOSURES}

Funding for this study was provided by Eli Lilly and Company and was obtained by authors C. Daniel Mullins and Robert L. Ohsfeldt, who serve as consultants to the company. Mullins served as principal author of the study. Study concept and design, analysis and interpretation of data, drafting and critical revision of the manuscript, and statistical expertise were contributed by both authors.

\section{REFERENCES}

1. Gold MR, Siegel JE, Russell LB, Weinstein MC. Cost-Effectiveness in Health and Medicine. New York: Oxford University Press;1996.

2. Lyles A, Luce BR, Rentz AM. Managed care pharmacy, socioeconomic assessments and drug adoption decisions. Soc Sci Med. 1997;45:513-24.

3. Grabowski H, Mullins CD. Pharmacy benefit management, cost-effectiveness analysis and drug formulary decisions. Soc Sci Med. 1997;45:537-46.

4. Ettinger B, Black DM, Mitlak BH, et al. Reduction of vertebral fracture risk in postmenopausal women with osteoporosis treated with raloxifene. JAMA. 1999;282:637-45.

5. Black DM, Cummings SR, Karpf DB, et al. Randomised trial of effect of alendronate on risk of fracture in women with existing vertebral fractures. Lancet. 1996;348:1535-41.

6. Maricic M, Adachi JD, Sarkar S, et al. Early effects of raloxifene on clinical vertebral fractures at 12 months in postmenopausal women with osteoporosis. Arch Intern Med. 2002;162:1140-43.

7. Cited in alendronate product information provided by manufacturer. Available at: http://www.merck.com/product/usa/fosamax/hcp/home.html.

8. National Osteoporosis Foundation. Osteoporosis: review of the evidence for prevention, diagnosis and treatment and cost-effectiveness analysis.

Osteoporosis Int. 1998;8(suppl 4):S1-S88.

9. Cummings, SR, Palermo L, Browner W, et al. Monitoring osteoporosis therapy with bone densitometry: misleading changes and regression to the mean. JAMA. 2000;283:1318-21.

10. NIH Consensus Development Panel on Osteoporosis Prevention, Diagnosis, and Therapy. Osteoporosis prevention, diagnosis, and therapy. JAMA. 2001;285,785-95

11. Delmas PD, Bjarnason NH, Mitlak BH, et al. Effects of raloxifene on bone mineral density, serum cholesterol concentrations, and uterine endometrium in postmenopausal women. N Engl J Med. 1997;337:1641-47.

12. Writing Group for the Women's Health Initiative Investigators. Risk and benefits of estrogen plus progestin in health postmenopausal women: principal results from the Women's Health Initiative randomized controlled trial.
JAMA. 2002;288:321-33.

13. Barrett-Connor E, Grady D, Sashegyi A, et al. Raloxifene and cardiovascular events in osteoporotic postmenopausal women: four-year results from the MORE randomized trial. JAMA. 2002;287:847-57.

14. Walsh BW, Kuller LH, Wild RA, et al. Effects of raloxifene on serum lipids and coagulation factors in healthy postmenopausal women. JAMA. 1998;279:1445-51.

15. Brown WV. Landmark trials in lipid reduction. Value Health. 1998;1:110-14. 16. Hulley S, Grady D, Bush T, et al. Randomized trial of estrogen plus progestin for secondary prevention of coronary heart disease in postmenopausal women. JAMA. 1998;280:605-13.

17. Blakely JA. The Heart and Estrogen/Progestin Replacement Study revisited: hormone replacement therapy produced net harm, consistent with the observational data. Arch Intern Med. 2000;160:2897-900.

18. Rossouw JE. Early risk of cardiovascular events after commencing hormone replacement therapy. Curr Opinion Lipidol 2001:12:371-75.

19. Cummings SR, Eckert S, Krueger KA, et al. The effect of raloxifene on risk of breast cancer in postmenopausal women. JAMA. 1999;281:2189-97.

20. Jordan VC, Glusman JE, Eckert S, et al. Incident primary breast cancers are reduced by raloxifene: integrated data from multicenter, double-blind, randomized trials in $\sim 12,000$ postmenopausal women. Abstract 466. In:

Program/proceedings of the 34th Annual Meeting of the American Society of Clinical Oncology; May 16-19, 1998; Los Angeles, CA.

21. Data on file, Eli Lilly and Company.

22. Final HERS data from HERS Web site. Available at: http://www.epibiostat.ucsf.edu/HERS/ptable5.html.

23. Collaborative Group on Hormonal Factors in Breast Cancer. Breast cancer and hormone replacement therapy: combined reanalysis of data from 51 epidemiological studies involving 52,705 women with breast cancer and 108,411 women without breast cancer. Lancet. 1997;350:1047-59.

24. Colditz GA. Relationship between estrogen levels, use of hormone replacement therapy, and breast cancer. J Natl Cancer Inst. 1998;90:814-23.

25. Ettinger B, Li DK, Klein R. Continuation of postmenopausal hormone replacement therapy: comparison of cyclic versus continuous combined schedules. Menopause. 1996;3(4):185-89.

26. Kayser J, Ettinger B, Pressman A. Postmenopausal hormone support: discontinuation of raloxifene versus estrogen. Menopause. 2001;8:328-32.

27. Price data from Analysource.com

28. Ettinger B, Selby JV, Citron JT, et al. Gynecologic complications of cyclic estrogen progestin therapy. Maturitas. 1993;17:197-204.

29. Ettinger B, Li DK, Klein R. Unexpected vaginal bleeding and associated gynecologic care in postmenopausal women using hormone replacement therapy: comparison of cyclic versus continuous combined schedules. Fertil Steril. 1998;69:865-69.

30. Gavin NI, Ohsfeldt RL, Thorp JM, Bray JW. Medical care costs associated with postmenopausal hormone replacement therapy. Osteoporosis Int. 2000;11(suppl 2):S180.

31. Ettinger B, Pressman A, Schein J, et al. Clinic visits and hospital admissions for care of acid-related upper gastrointestinal disorders in women using alendronate for osteoporosis. Am J Managed Care. 1998;4:1377-82.

32. Colby CJ, Levin TR, Boyko WL, Schein JR. Costs of Acid-related Disorders Associated With Alendronate Use for Osteoporosis in a Large Managed Care Organization. Paper presented at: 21st Annual Meeting of the American Society for Bone and Mineral Research; September 30-October 4, 1999; St. Louis, MO.

33. Data on file, Eli Lilly and Company.

34. Data on file, Eli Lilly and Company.

35. Office of Technology Assessment (OTA). Effectiveness and Costs of Osteoporosis Screening and Hormone Replacement Therapy. Washington, DC: Government Printing Office; 1995. 
36. Legorreta AP, Brooks RJ, Leibowitz AN, et al. Cost of breast cancer treatment: a 4-year longitudinal study. Arch Intern Med. 1996;156:2197-201.

37. U.S. Preventive Services Task Force. Postmenopausal hormone replacement therapy for primary prevention of chronic conditions: recommendations and rationale. Ann Intern Med. 2002;137:834-39.

38. Armstrong K, Chen T-M, Albert D, et al. Cost-effectiveness of raloxifene and hormone replacement therapy in postmenopausal women: impact of breast cancer risk. Obstet Gynecol. 2001;98:996-1003.

39. Iglesias CP, Torgerson DJ, Bearne A, Bose U. The cost utility of bisphosphonate treatment in established osteoporosis. Q J Med. 2002;95:305-11.

40. Tosteson AN, Weinstein MC. Cost-effectiveness of hormone replacement after the menopause. Baillieres Clin Obstet Gynecol. 1991;5:943-59.

41. Barrett-Connor E, Grady D, Sashegyi A, Cox DA, Harper KD. Raloxifene and risk of stroke among high-risk women in the Multiple Outcomes of

Raloxifene Evaluation (MORE) randomized trial. Abstract 3555. Paper presented at: American Heart Association National Meeting; November 17, 2002; Orlando, FL. 